\title{
CAMBIO CLIMÁTICO, MOVILIDAD HUMANAY SU IMPACTO EN LAS RELACIONES INTERNACIONALES DEL SIGLO XXI
}

\author{
CLIMATE CHANGE, HUMAN MOBILITY AND ITS IMPACT ON \\ INTERNATIONAL RELATIONS OF THE 21ST CENTURY
}

\author{
Juan Carlos Méndez Barquero ${ }^{1}$
}

\section{RESUMEN:}

El artículo aborda los principales retos que implica la movilidad humana en el contexto de los impactos adversos del cambio climático en las relaciones internacionales contemporáneas, en especial desde la perspectiva de los vacíos legales y operativos que existen para abordar las necesidades de las personas que se ven forzadas a cruzar una frontera internacional por un desastre repentino o eventos de evolución lenta. Se examinan algunos marcos e iniciativas internacionales, prácticas eficaces y algunos retos en relación con el fenómeno multidimensional de la movilidad humana en el contexto del cambio climático, la cual, según la evidencia científica contemporánea, demuestra que los desastres y los efectos del cambio climático desplazaron un promedio de 26 millones de personas cada año entre 2008 y 2015.

Palabras clave: Migración; movilidad humana; cambio climático; desastres; vacíos internacionales.

\begin{abstract}
:
This paper addresses the major challenges of human mobility in the context of the adverse impacts of climate change on contemporary international relations, especially from the perspective of legal and operational gaps that exist to address the needs of people who are forced to cross an international border by a sudden disaster or slow onset events. To this end, the paper examines some frameworks and international initiatives, effective practices and some challenges related to the multidimensional phenomenon of human mobility in the context of climate change, which current scientific evidence shows that disasters and the effects of climate change displaced an average of 26 million people each year between 2008 and 2015.
\end{abstract}

Key words: migration; human mobility; climate change; disasters; international gaps

1 Profesor de derechos humanos y derecho internacional humanitario en la Escuela de Relaciones Internacionales de la Universidad Nacional, Costa Rica. Correo electrónico: juan.mendez.barquero@, una.cr 


\section{Introducción}

En la última década, la comunidad internacional ha experimentado un creciente interés político y científico sobre el fenómeno del desplazamiento en el contexto de desastres y, particularmente, en relación con los efectos adversos del cambio climático.

Las inundaciones en Pakistán en 2010, que afectaron hasta el 20 por ciento del país y que dejaron un estimado de 20 millones de personas temporalmente sin hogar; las grandes inundaciones de principios de 2011 en el este de Australia, que dejaron a muchos pueblos hundidos durante varias semanas, son eventos que capturaron no solo la atención de los medios de comunicación a nivel internacional, sino que contribuyeron al debate sobre las consecuencias desastrosas y visibles de la interacción entre la movilidad humana y los cambios ambientales de evolución lenta y repentina (Warner, 2011).

Este creciente interés de la comunidad científica y de quienes toman las decisiones a nivel internacional ha sido matizado por un abordaje multidimensional, constituyéndose la movilidad humana hoy en día -en el contexto del cambio climático- en una temática de la más alta relevancia en los foros internacionales más importantes como la misma Convención Marco de Naciones Unidas sobre Cambio Climático (UNFCCC por sus siglas en inglés), el Panel Intergubernamental sobre Cambio Climático (IPCC por sus siglas en inglés), el Marco de Sendai sobre Reducción del Riesgo de Desastres, el Consejo de Derechos Humanos de las Naciones Unidas, entre otros.

Por otro lado, las dos agencias internacionales de mayor peso político y trayectoria técnica en relación con el trabajo con poblaciones refugiadas y migrantes, el Alto Comisionado de las Naciones Unidas para los Refugiados (ACNUR) y la Organización Internacional para la Migraciones (OIM), cuentan hoy con líneas de trabajo claras en cuanto a dicha temática o unidades especializadas en el tema. En el caso de la OIM, por ejemplo, la nueva División sobre Migración, Ambiente y Cambio Climático fue creada a inicios del año 2014 para abordar las amenazas y retos de lo que por años han denominado "migraciones ambientales".

Sin embargo, no fue sino hasta diciembre de 2010 cuando la comunidad internacional logró el reconocimiento político de uno de los mayores acuerdos en materia de movilidad humana y cambio climático, la incorporación del párrafo 14 (f) en el Marco de Adaptación de Cancún, que fue aprobado durante las negociaciones de la Convención Marco de las Naciones Unidas sobre Cambio 
Climático número diez y seis (COP16). En dicho acuerdo se insta a los Estados parte de dicha Convención a:

La adopción de medidas para mejorar el entendimiento, la coordinación y la cooperación en lo que respecta al desplazamiento, la migración y el traslado planificado como consecuencia del cambio climático, cuando corresponda, a nivel nacional, regional e internacional (UNFCCC, 2011).

Este se convirtió en el primer reconocimiento legal en un acuerdo internacional vinculante relativo a las interacciones entre cambio climático y la migración (Betts, 2014). Sin embargo, años más tarde, la adopción del 'Acuerdo de París' en 2015 vuelve a reafirmar este compromiso cuando 195 Estados parte de la UNFCCC acuerdan -entre otros temas- evitar, minimizar y abordar el desplazamiento asociado a los efectos adversos del cambio climático a través de la conformación de un task force sobre la materia asesor al Mecanismo de Varsovia sobre daños y pérdidas (Naciones Unidas, 2015).

A la COP del año 2010, le siguieron una serie de eventos internacionales de alta relevancia sobre la materia y otras reuniones de alto nivel, como, por ejemplo, la celebración de una reunión de personas expertas en Bellagio, Italia, en febrero de 2011, la Conferencia Nansen sobre Cambio Climático y Desplazamiento en el siglo 21 que tomó lugar en Oslo en junio de 2011, organizada por el Ministerio Noruego de Medio Ambiente y el Ministerio de Asuntos Exteriores, en cooperación con el Consejo Noruego para los Refugiados, y otros espacios que fueron dando impulso a la creación de un nuevo consenso internacional sobre el abordaje multidimensional de esta de esta temática.

\section{La migración en el contexto de cambio climático como reto multidimensional}

Si bien la movilidad humana en el contexto de desastres y cambio climático ha sido entendida como una respuesta y estrategia de adaptación natural del ser humano, a nivel internacional este desplazamiento de personas (interno y transfronterizo) tiene impacto mayor, en términos políticos, de protección internacional y en relación con los impactos económicos asociados.

El derecho internacional, por ejemplo, solo reconoce una pequeña clase de los grupos migrantes forzosos como personas a las que un tercer país tiene la obligación de proteger, es decir, personas refugiadas o apátridas. El desplazamiento transfronterizo como consecuencia de los desastres naturales y los efectos del 
cambio climático, por tanto, ha sido identificado como un vacío normativo en la protección internacional (McAdam, 2011).

La movilidad humana en el contexto del cambio climático ha tenido, además, un impacto directo sobre los temas de alta política, tales como seguridad e implicaciones geopolíticas asociadas. Entre las principales amenazas identificadas se encuentran las migraciones por cuestiones ambientales -especialmente las provenientes de África- y el debate actual sobre las consecuencias devastadoras que podría causar un aumento en el nivel del mar en los pequeños Estados insulares en el Pacífico.

En relación con las migraciones por cuestiones ambientales, informes recientes de la Organización Internacional para las Migraciones (OIM) establecen con ejemplos actuales, el nexo y las complejas relaciones entre cambio climático, degradación ambiental; y como todo ello conlleva a movimientos migratorios importantes entre naciones, especialmente en el continente africano y el sudeste de Asia. En este sentido, la OIM indica:

...Esencialmente, hay una relación de dos vías entre la migración y el medio ambiente: los factores ambientales precipitan la migración y la migración afecta al medio ambiente. El cambio climático añade una nueva complejidad a este nexo al exacerbar la degradación ambiental ... (OIM, 2009, p. 2)

Este panorama político se complica aún más cuando los estudios científicos y estadísticas demuestran, por ejemplo, que los desastres provocados por amenazas naturales han obligado a millones de personas a huir de sus hogares cada año en todas las regiones el mundo. Al respecto, el Centro de Monitoreo sobre del Desplazamiento Interno del Consejo Noruego para Refugiados (IDMC por sus siglas en inglés) afirma que el desplazamiento causado por el cambio climático y los desastres repentinos es un fenómeno global, y su escala cada vez mayor, la frecuencia y la complejidad plantean enormes desafíos para las poblaciones expuestas y vulnerables, gobiernos, retos humanitarios y desarrollo de las organizaciones (IDMC, 2015).

Este mismo centro de investigación científica en su último estudio demuestra que los desastres - muchos de ellos asociados a fenómenos meteorológicos y variabilidad climático- desplazaron un promedio de 26,4 millones de personas cada año entre 2008 y 2015 (IDMC, 2015).

Asimismo, para el 2008, un informe sobre el cambio climático y la seguridad internacional presentado por el Alto Representante de la Unión Europea para la Política Exterior y Seguridad Común, en conjunto con la Comisión Europea, 
identifica siete amenazas básicas que se ciernen sobre la humanidad, como consecuencia del calentamiento global: conflictos por los recursos, daños y riesgos económicos para las ciudades costeras y las infraestructuras vitales; pérdida de territorio y contenciosos fronterizos; migraciones por causas ambientales; situaciones de fragilidad y radicalización; tensiones por el suministro de energía, y presiones sobre la gobernanza internacional (European Commission, 2008).

\section{Migraciones y desplazados ambientales: Entre la realidad y la prospectiva}

No existe hoy un término internacionalmente aceptado para el movimiento de personas por razones climatológicas o ambientales. Las personas que migran por motivos ambientales no entran dentro de ninguna categoría en particular prevista por el derecho internacional moderno. Por ejemplo, términos como "refugiado ambiental", "refugiado climático", no tienen base legal dentro del derecho internacional de los grupos refugiados ${ }^{2}$, por lo cual el consenso entre las organizaciones internacionales que trabajan en la materia, tales como la OIM y el ACNUR, es evitar, inclusive, el uso de estos conceptos para no incurrir en errores de tipo legal.

En un intento por contribuir al debate de este complejo y amplio fenómeno, la Organización Internacional de las Migraciones (IOM) ha avanzado en una definición sobre los migrantes medioambientales:

...Migrantes ambientales son personas o grupos de personas, quienes predominantemente por cambios espontáneos o progresivos en el ambiente que afectan sus vidas y condiciones de vida, eligen o son obligados a dejar sus hogares temporal o permanentemente y quienes se desplazan tanto dentro de su propio país, como fuera... (IOM, 2009, p. 5)

Si bien la definición anterior es bastante integral, una clara distinción entre las migraciones voluntarias y obligadas, debido a factores ambientales, es difícil de establecer, con la excepción quizá de los desastres naturales.

McAdam y Limon (2015) entienden el desplazamiento como un fenómeno que describe el movimiento forzado de personas de sus hogares. Puede ser, tempo-

2 La Convención sobre el Estatuto de los Refugiados de 1951 establece claramente que una persona refugiada es aquella que debido a persecución o a un temor fuertemente fundamentado de persecución por razones de raza, religión, nacionalidad, membresía en un grupo social en particular u opinión política, huye de su país natal para refugiarse en un tercer país. 
ral o permanente, a pequeña o en gran escala, y puede ocurrir dentro de un país o a través de una frontera internacional.

El tema de las personas desplazadas y migraciones inducidas por los efectos del cambio climático global es una de las principales preocupaciones de los actores estatales. La lógica de la migración es básica, se presume que el daño causado por las tormentas y el incremento en los niveles del mar, especialmente en las áreas costeras, el Caribe y los pequeños Estados insulares, incrementará las migraciones desde estos países, hacia los países mejor adaptados a las consecuencias climáticas, como los Estados Unidos y los países europeos.

Según estimaciones recientes, algunas regiones en particular como el sudeste asiático, África y Europa presentarían retos geopolíticos asociados al aumento de la movilidad humana por cuestiones relativas al cambio ambiental y la frecuencia de desastres, aunque otros afirman que ninguna otra región será tan afectada directamente por las migraciones climáticas, como la región del sudeste asiático $^{3}$ (Podesta \& Ogden, 2007).

El caso de la población de Bangladesh, que se estima en unos 142 millones de personas, se prevé que aumentará en unos 100 millones más durante las próximas décadas, aun cuando el impacto del cambio climático y otros factores ambientales hará que las regiones bajas y costeras de ese país sean inhabitables (Podesta \& Ogden, 2007).

Gran cantidad de los grupos desplazados se moverán hacia el interior del país, lo cual fomentará la inestabilidad en la población reasentada, por la competencia por los ya de por sí escasos recursos de la población residente establecida. Por la imposibilidad de establecer un nivel de vida estable y digno, decenas de miles bangladeshís han emigrado. Desde la década de 1950, entre 12 y 17 millones de bangladeshís migraron a la India, atraídos por un mayor nivel de vida y una baja densidad de población. Miles de árboles se talaron para emprender prácticas agrícolas, y ello resultó en una terrible erosión del suelo y medios de vida sostenibles locales (Smith \& Vivekananda, 2007).

En general, algunos de los movimientos serán entre una comunidad rural hacia otra, por aquellos grupos que esperan mantener su estilo de vida anterior. Otros movimientos serán desde las áreas rurales donde el estilo de vida agrícola ha

3 Se ha estimado que alrededor de un $40 \%$ de la población de Asia habita a $60 \mathrm{Km}$ de las zonas costeras, por lo que una subida estrepitosa del nivel del mar puede amenazar el hábitat de millones de personas. La tensión producida por la escasez de agua y la pérdida de productividad agrícola harán muy difícil la sostenibilidad alimenticia de la región. 
sido afectado por el cambio climático, hacia centros urbanos donde se espera encontrar mejores opciones de calidad de vida. Muchos cruzarían las fronteras de sus países, esperando una mayor oportunidad de desarrollo. Una parte significativa de estas tendencias de la migración global acelerará la urbanización, agregando pobreza urbana, conflictos locales y, posiblemente, criminalidad.

Algunos estudios proyectan que miles intentarán migrar al extranjero, y crearán tensión política, entre los grupos pobladores originarios y los nuevos vecinos. Norman Myers de la Universidad de Oxford y el 'Informe Sterns' sobre las consecuencias económicas del cambio climático han sugerido que hasta 200 millones de personas podrían movilizarse debido a factores ambientales para el año 2050 (IOM, 2009, p. 1).

En África, Nigeria es un país que eventualmente sufriría una terrible sequía inducida por el cambio climático, la cual, sumada a la desertificación del país, la elevación del nivel del mar y un elevado crecimiento demográfico, obligará a cientos de personas a emigrar de las zonas costeras, lo que contribuirá a exacerbar los conflictos internos existentes sobre la producción de petróleo en el Delta del Níger (Podesta \& Ogden, 2007, p. 57).

Otro ejemplo que ha suscitado mucha atención a nivel internacional es el caso de Darfur, en Sudán, región en donde la escasez de agua y la desertificación de grandes extensiones de tierras de cultivo y pastizales ha generado, en los últimos años, una feroz competencia entre grupos agricultores y ganaderos por el acceso a tierra cultivable, la cual, combinada con tensiones étnicas y religiosas, ha contribuido a exacerbar un nivel de violencia tal, que la misma Corte Penal Internacional decretó, en el año 2008, una orden de captura internacional al presidente de ese país y varios líderes tribales, por crímenes de guerra y genocidio ${ }^{4}$.

El impacto del cambio climático en Sudán, en particular, los veinte años de sequía en la zona de Sahel, desempeñó un papel importante en la intensificación de las quejas y tensiones internas, ya que significaba que había menos tierra disponible, tanto para la agricultura, como para el pastoreo. Estas situaciones propiciaron negativamente un ambiente de marginación económica y política, así como de violencia física. El número de conflictos violentos atribuibles a las controversias tradicionales sobre el uso de la tierra aumentó dramáticamente a partir de la década de los años 1970, y en especial a mediados de la década de 1980, cuando la guerra civil entre el norte y el sur de Sudán estalló, y el gobier-

4 Aunque no se podría afirmar que esta decisión de la Corte Penal Internacional se deba estrictamente a tensiones y violencia causada por motivos ambientales. 
no utilizó milicias tribales árabes como un medio de mantener controlados a los rebeldes del sur (Smith \& Vivekananda, 2007, p.12).

Así, el cambio climático por sí solo no explica el brote o la extensión de la violencia en Darfur. Al menos otros dieciséis países de la zona del Sahel en África han sentido el impacto del calentamiento global, incluidos Malí y El Chad, pero solo Sudán ha experimentado un conflicto tan devastador, exacerbado directamente por factores ambientales.

El desplazamiento masivo de personas en Darfur conlleva asimismo un serio impacto en el medio ambiente. Campamentos temporales para personas desplazadas se traducen en cientos de árboles talados para leña y consumo doméstico. El consumo de árboles es mayor en ciertos campamentos donde las fábricas de ladrillos, que son la única fuente de subsistencia para estas personas, requieren hasta 200 árboles por día para sostener la producción (Smith \& Vivekananda, 2007). Con el paso de las semanas y los meses, estos dos factores combinados han producido un nivel de deforestación insostenible en las regiones donde se encuentran estos campamentos.

Hacia inicios de los años 1990, la migración rural se había convertido en un fenómeno estructural, por ejemplo, en la región senegalesa de Tambacounda, afectada por una severa erosión del suelo, producto de este fenómeno el $90 \%$ de los hombres de la región entre los 30 y 60 años habían migrado al menos una vez en sus vidas (IOM, 2009, p. 3).

Por otro lado, cuando la degradación ambiental se vuelve severa e irreversible, la migración resultante se vuelve asimismo permanente y requiere una reubicación de las poblaciones afectadas, como ocurrió en el caso de Brasil, en donde entre 1970 y 2005, unos 60 millones de personas migraron de las áreas rurales a las zonas urbanas de ese país, especialmente desde la región de Sertão, repetidamente golpeada por períodos de sequía y épocas secas que afectaron prácticamente todas las actividades agrícolas, en especial a los pequeños y medianos campesinos. La escasez de recursos ha llevado, además, a la gente a trasladarse temporal o definitivamente a otros lugares. En Irán, al oeste de la India y Pakistán, y en el norte y el oeste de China, numerosas aldeas se han despoblado debido a la falta de recursos hídricos (Brown, 2005, citado por GTZ, 2008).

En Europa, tal y como lo señaló en el año 2008 el Alto Representante de la Unión Europea para la Política Exterior y la Seguridad Común y la Comisión Europea, las migraciones por causas ambientales, que según proyecciones de las Naciones Unidas podrían llegar a ser millones hacia el año 2020, pueden incrementar los conflictos en las zonas de tránsito y de destino y, por lo tanto, 
Europa debe disponerse a sufrir una presión migratoria considerablemente mayor (European Commission, 2008).

Por otro lado, para el Consejo Asesor Alemán sobre Cambio Climático, las distintas fases y elementos que contribuyen a las migraciones inducidas por cuestiones ambientales incluyen la etnicidad, la estabilidad política, la estructura gubernamental, así como el eventual desencadenamiento en conflicto y tensiones. Este esquema es central para comprender las preocupaciones de la política europea, pues un notable aumento en la migración inducida por el medio ambiente supone que la migración podría ser un desencadenante de conflictos violentos (GIZ, 2008). La degradación gradual del agua y la tierra y, por consiguiente, la decisión de migrar, pueden conducir a la desestabilización y generar conflictos en las regiones de origen, tránsito o destino.

Por otro lado, los elementos religiosos y étnicos podrían eventualmente jugar un papel central en las dinámicas migratorias, pues, para un sector, el número de personas migrantes musulmanas en la Unión Europea podría exacerbar las tensiones existentes e incrementar la radicalización entre las crecientes comunidades islámicas débilmente asimiladas en Europa.

\section{Vacíos de protección y la necesidad de un nuevo marco internacional}

Como se explicó antes, no existe un marco internacional vinculante relacionado con la protección de las personas que cruzan una frontera internacional por causa de un desastre repentino como puede ser un terremoto, una inundación, o bien un evento de evolución lenta asociado al cambio climático. Al respecto, Volker Türk, Alto Comisionado Adjunto para los Refugiados de las Naciones Unidas, explica que no existe un instrumento internacional en la actualidad que proteja a las personas que se desplazan a través de las fronteras como consecuencia del cambio en el clima. Si, como se espera, el desplazamiento transfronterizo en el contexto de los desastres y del cambio climático aumenta, las brechas que existen sobre la protección de las personas desplazadas en estos contextos serán más prominentes (Türk, 2015).

El marco internacional sobre la protección de personas refugiadas adoptado durante la segunda mitad del siglo XX no contempla el factor ambiental ni mucho menos las amenazas del cambio climático como motivo fundado para solicitar el estatus y condición de sujeto refugiado. 
Si bien numerosos artículos periodísticos e inclusive libros que abordan la temática se refieren a conceptos como "refugiados climáticos" (Altamirano, 2013), este es un término incorrecto a la luz del derecho internacional público y más bien podría generar confusión.

Mc Adam (2011) explica que hasta ahora ha existido un número reducido de casos en Australia y Nueva Zelanda, donde personas de Tuvalu y Kiribati han tratado de argumentar que deben recibir protección internacional en calidad de refugiados por los impactos del cambio climático. Otros grupos solicitantes de Tonga y Bangladesh han buscado protección sobre la base de los desastres naturales repentinos.

En Nueva Zelanda, por ejemplo, las resoluciones judiciales no han favorecido a quienes han argumentado la necesidad de protección internacional por causa de los impactos del cambio climático. Los tribunales de ese país han argumentado que no se está ante un derecho de los recurrentes similar a los daños y persecución que incluye la Convención o de los Refugiados (Mc Adam, 2011).

Es esta brecha legal internacional la que ha servido de plataforma justamente para la creación de alianzas internacionales y otras iniciativas como la "Iniciativa Nansen" para la creación de consensos internacionales sobre las necesidades de protección.

Al respecto, quizá la iniciativa global de mayor proyección internacional sobre la materia es justamente la "Iniciativa Nansen", un proceso intergubernamental creado en el año 2012 para generar un consenso internacional sobre las necesidades de protección de las personas que se van forzadas a cruzar una frontera internacional en el contexto de desastres y efectos del cambio climático.

Esta iniciativa internacional, que concluyó en su primera etapa en octubre de 2015, fue liderada por una copresidencia compartida entre Noruega y Suiza y respaldada a nivel internacional por Alemania, Australia, Costa Rica, México, Bangladesh, Filipinas y Kenia. En palabras del mismo Enviado de la Iniciativa Nansen:

El propósito de la Iniciativa Nansen fue construir un consenso entre los Estados afectados sobre cómo responder adecuadamente a los retos del desplazamiento transfronterizo en el contexto de desastres, incluidos los efectos del cambio climático. (Kälin, 2015, p. 5)

Hasta la fecha, los esfuerzos de esta coalición internacional cuya Secretaría Técnica y Enviado Especial se ubicó en Ginebra, Suiza, ha sido apoyada al más alto nivel político tanto por el ACNUR como por la OIM, y dio como resultado 
la adopción de lo que se denomina una 'Agenda de protección para la protección de personas desplazadas a través de fronteras en el contexto de desastres y cambio climático' (The Nansen Initiative, 2015).

Este documento -que no es vinculante- reúne las prácticas más eficaces, herramientas y experiencias recopiladas a través de la realización de más de diez consultas regionales intergubernamentales, sociedad civil y academia entre los años 2012 y 2015 en América Central y América del Sur, África del Sur, Sudeste Asiático, Asia del Sur, Gran Cuerno de África y el Pacífico.

A estas iniciativas se suman otras como por ejemplo la Iniciativa "Migrants in Countries in Crisis MICIC" (Migrantes en países en Crisis), una iniciativa internacional de la Organización Internacional para las Migraciones (OIM) que adopta una metodología similar a la Iniciativa Nansen y que comparte un ámbito de acción en relación con las necesidades de protección de migrantes que se encuentran en tránsito o residiendo en un tercer país que es afectado por un desastre causado por una amenaza natural.

Por otro lado, estas necesidades de protección se ven exacerbadas cuando el cambio climático se convierte justamente en un causante de conflictos. Al respecto, el informe del IPCC también considera que el cambio climático puede aumentar indirectamente riesgos de conflictos violentos en la forma de una guerra civil y la violencia entre los grupos, mediante la amplificación, bien documentada, de conductores de estos conflictos, como la pobreza y las crisis económicas.

Múltiples líneas de evidencia relacionan la variabilidad climática a estas formas de conflicto y el desplazamiento forzado de personas. Para el ACNUR hay un caso de protección de los sujetos refugiados en situaciones donde la acción perjudicial o inacción de un gobierno en el trato con los eventos relacionados con el clima se relaciona con uno o más de los motivos de la Convención de Refugiados (como la negación de la asistencia humanitaria a un grupo minoritario) y podría, por lo tanto, considerarse persecución (ACNUR, 2014).

Por otro lado, generalmente es difícil aislar los factores climáticos y ambientales como el único conductor de los movimientos de población. Como ejemplo, la mayor afluencia de somalíes en Kenia en 2012 y 2013 fue motivada tanto por la sequía como por la hambruna que afecta a Somalia.

La comunidad internacional también ha pedido en repetidas ocasiones que los principios de derechos humanos se integren en la estructura actual de las negociaciones sobre cambio climático, con el fin de fortalecer las respuestas ante 
las poblaciones más vulnerables, incluidas migrantes y personas que se van forzadas a desplazarse entre fronteras.

Tal es el caso de Consejo de Derechos Humanos de las Naciones Unidas, uno de los órganos políticos más relevantes en materia de derechos humanos de ese organismo internacional, el cual en junio de 2015 adoptó una resolución sobre cambio climático y derechos humanos afirmando que las obligaciones, estándares y principios de los derechos humanos tienen el potencial para informar y fortalecer la formulación de políticas a nivel regional y nacional en el ámbito del cambio climático (Human Rights Council, 2015).

Tal y como afirman McAdam y Limon (2015) lo que está claro es que el Consejo de Derechos Humanos, por sí solo no será capaz de cerrar la brecha de protección de los derechos humanos para las personas desplazadas través de las fronteras en el contexto de los desastres, pero sí emite un llamado político al más alto nivel, con el cual, con un apropiado desarrollo de políticas e iniciativas conexas, tales como la Iniciativa Nansen, se podrá abordar, seriamente, la mejor manera de proteger a estas personas en tales situaciones.

Es importante indicar que en estos esfuerzos trabajan actualmente tanto el mismo Alto Comisionado de las Naciones Unidas para los Refugiados (ACNUR), apoyando sistemáticamente el desarrollo de investigación y recolección de datos empíricos sobre la materia; la Organización Internacional para las Migraciones (OIM), a través de su Unidad sobre Migración, Ambiente y Cambio Climático, con esfuerzos importantes en el desarrollo de investigación sobre la temática, documentos de política pública y lineamientos sobre el abordaje de la movilidad humana como estrategia de adaptación al cambio climático, gestión y buen gobierno de las migraciones y gestión fronteriza, entre otros.

Importante destacar, en este sentido, que la OIM recientemente lazó por primera vez un Atlas de las Migraciones Ambientales, construido sobre la base de los conocimientos, datos y estudios de casos producidos en los últimos años por la OIM y otras organizaciones internacionales, instituciones académicas y grupos investigadores de todo el mundo, como una herramienta innovadora que proporciona una descripción visual de esta tendencia de la migración humana a través de mapas, ilustraciones y textos explicativos elaborados con la supervisión de personal experto mundial en este campo (IOM, 2015).

Asimismo, es importante indicar que desde principios de la década de1990, la OIM ha trabajado activamente en el ámbito de la migración, el cambio climático y el medio ambiente, y ha llevado a cabo programas para hacer frente a este desafío en más de 40 países en el Pacífico, América Latina, Asia y África. En 
muchas de estas áreas, la organización ha colaborado con personas afectadas por huracanes, inundaciones y sequías (IOM, 2009).

Desde las devastadoras consecuencias del huracán Mitch en Honduras (1998) hasta los ciclones que han afectado cientos de personas en Madagascar, esta organización internacional con el concurso de otras decenas de organizaciones no gubernamentales han apoyado los esfuerzos gubernamentales para hacer frente a los movimientos de población forzados por eventos extremos asociados con la variabilidad climática, como el diseño de programas de reasentamiento planificado, asistencia humanitaria y protección a los grupos desplazados.

Sin embargo, es necesario indicar que si bien no existe un marco internacional vinculante en cuanto a la protección de personas que atraviesan una frontera de manera forzada ante un evento o amenaza natural, por años muchos países han implementado medidas de protección -en muchos casos de manera temporalpara la protección de estas personas.

Al respecto, la Iniciativa Nansen ha identificado que:

Por lo menos 50 países que en décadas recientes han acogido a personas tras la ocurrencia de desastres o que se han abstenido de devolverlas; especialmente en casos de desastres ocasionados por tormentas tropicales, inundaciones, sequías, tsunamis y terremotos. ... Debido a la falta de un monitoreo sistemático de los desplazamientos a través de fronteras en el contexto de desastres, esta cifra dista mucho de ser completa. Los datos mundiales que están disponibles actualmente abarcan únicamente la incidencia del desplazamiento y no los lugares hacia donde huyen las personas desplazadas o donde se asientan finalmente. (The Nansen Initiative, 2015, p. 1)

Por ejemplo, un día después del terremoto en Haití, el Departamento de Seguridad Nacional de Estados Unidos anunció la implementación de "Estatus de Protección Temporal" (TPS por sus siglas en inglés) para detener la deportación de haitianos a su país de origen. Cabe destacar que Estados Unidos ha concedido la condición de TPS en numerosas ocasiones, tanto para emergencias políticas y por motivos de desastres naturales (Fagen, 2013). El caso más reciente de este tipo de implementación de medidas de protección temporal se dio en el contexto del terremoto que afectó Nepal en 2015, en donde Estados Unidos aplicó el mismo sistema de TPS asegurando la estancia de nacionales de Nepal en Estados Unidos por un período de un año y garantías conexas como oportunidades para aplicar a empleos. 
La región latinoamericana, por otro lado, cuenta con prácticas muy interesantes en este sentido. Países como Panamá o Argentina cuentan con legislación migratoria que permite que la discrecionalidad se aplique en situaciones similares o por urgencia humanitaria. Otros países como Perú ya cuentan con legislación humanitaria o la existencia de "visas humanitarias" que otorgan medidas de protección a personas que se ven obligadas a huir de sus países por un desastre causado por una amenaza natural.

Durante el huracán Mitch que afectó la región centroamericana, pero en especial a Honduras en el año 1998, países como Panamá y Costa Rica emitieron directrices ejecutivas para no expulsar o deportar ciudadanos de Honduras debido a la situación que atravesaba ese país.

Estas y muchas otras prácticas han sido sistematizadas por la Iniciativa Nansen y con la adopción de la "Agenda para la protección de las personas desplazadas a través de fronteras en el contexto de desastres y cambio climático", la cual, como se indicó antes, ha identificado por lo menos 50 países que en décadas recientes han acogido a personas tras la ocurrencia de desastres o que se han abstenido de devolverlas, especialmente en casos de desastres ocasionados por tormentas tropicales, inundaciones, sequías, tsunamis y terremotos (The Nansen Initiative, 2015).

Este documento integra, entre otros aspectos, la protección de personas desplazadas a través de fronteras en el contexto de desastres, gestión del riesgo de desplazamiento en el país de origen, reducir vulnerabilidades y fortalecer la resiliencia ante los riesgos de desplazamiento, migración en condiciones de dignidad, reubicación planificada, asistencia para personas desplazadas internas, ámbitos prioritarios de actuación futura, fortalecer la gestión del riesgo de desplazamiento en el país de origen, entre otros.

Sin embargo, el análisis de legislación, instituciones competentes y respuestas operativas relacionadas con la protección y asistencia para personas desplazadas a través de fronteras en el contexto de desastres revela que, en general, existe una falta de preparación y, en la mayoría de los casos, esto da lugar a respuestas ad hoc (Nansen Initiative, 2015).

\section{Consideraciones finales}

La comunidad internacional se encuentra en un momento propicio para lograr acuerdos en materia del desplazamiento en el contexto de cambio climático. Si bien el fenómeno implica un abordaje multidimensional, político, operativo y 
en estricto sentido humanitario, la inminencia de esta realidad no puede esperar muchos años.

Nos encontramos ante un panorama positivo de nuevos y remozados marcos institucionales internacionales dentro de los cuales esta realidad puede y debe ser abordada: la Agenda 2030, la Cumbre Mundial Humanitaria que organiza la Oficina de las Naciones Unidas para Asuntos Humanitarios (OCHA), el nuevo Marco de Sendai para la reducción del riesgo de desastres 2015-2030, el Foro de los Estados Insulares del Pacífico, los procesos subregionales como el Proceso Cartagena+30 sobre refugiados en América Latina y las negociaciones internacionales sobre cambio climático, entre muchos otros.

La comunidad científica, organismos internacionales y academia han contribuido con un enorme volumen de investigación especializada que ha evidenciado la necesidad de tomar medidas políticas y legales. Es claro que la variabilidad climática, sumada a otro conjunto de factores como pobreza, crecimiento poblacional, entre otros, han afectado y seguirán afectando de diversas maneras la migración humana y los desplazamientos forzados en todo el mundo. Los desastres de origen natural y los cambios en los patrones climáticos que pueden traer derretimiento de los glaciares, aumento del nivel del mar y la desertificación son y serán cada vez factores que incidirán en la migración y el desplazamiento.

Hasta la fecha, las respuestas han sido intermitentes, variadas y ad hoc, es decir, delimitadas temporalmente para el evento en particular, en tanto el sistema internacional moderno, las regulaciones sobre la condición del estatuto de personas refugiadas y otros marcos subregionales no contengan disposiciones claras y específicas para abordar esta realidad.

Al decir de Jan Egeland, secretario General del Consejo Noruego para Refugiados y anterior subsecretario General de las Naciones Unidas para Asuntos Humanitarios, el desplazamiento de desastres es un fenómeno con implicaciones para las principales áreas de la política mundial y la acción, actualmente en discusión. Su éxito dependerá de la medida en que se proporcione un marco coherente e integrado y de largo plazo. Sus resultados dependen, en gran medida, de la capacidad de los gobiernos para medir y demostrar avances concretos hacia el logro de estos objetivos. 


\section{Referencias}

Altamirano, T. (2013). Refugiados ambientales. Cambio climático y migración forzada. Perú: Fondo Editorial, Pontificia Universidad Católica del Perú.

Betts, A. (Febrero, 2014). The post-Nansen agenda: governing human mobility in the context of natural disasters and climate change. Ocassional Policy Paper, Oxford: University of Oxford.

Department of Homeland Security. (2015). DHS Announces Temporary Protected Status Designation for Nepal. Disponible en http://www.uscis.gov/news/dhsannounces-temporary-protected-status-designation-nepal

Egeland, J. (2014). Millions displaced by disasters no time to lose. Perspective, 02, 2015, 06-07.

Fagen, P. (2013) Receiving Haitian migrants in the context of the 2010 earthquake. Discussion paper Ginebra: IDMC-IDMC-NRC.

GTZ. (2008). Climate Change and Security. Challenges for German Development Cooperation. Alemania: Federal Ministry for Economic Cooperation and Development. Deutsche Gesellschaft für Technische Zusammenarbeit (GTZ).

European Commission. (2008). Climate change and international security. Paper from the High Representative and the European Commission to the European Council, S113/08 14 March. Bruselas: EU.

Human Rights Council. (2015). Human Rights and climate change. Disponible en http://www.ohchr.org/EN/Issues/HRAndClimateChange/Pages/HRClimateChangeIndex.aspx

IDMC. (2015). Global Estimates 2015. People displaced by disasters. Ginebra: NRC.IDMC.

IOM. (2009). Compendium of IOM's activities in Migration, Climate change and the environment. Ginebra: International Organization for Migration.

IOM. (2009). Migration, Climate Change and the Environment. Policy Brief, May 2009, Ginebra, Disponible en www.iom.int

IOM. (2015). Environmental Migration Portal. Disponible en http://www.environmentalmigration.iom.int/human-mobility-cop21

Kälin, W. (Mayo, 2015). The Nansen Initiative: building consensus on displacement in disaster contexts. Forced Migration Review, 49, 5-7.

Naciones Unidas. (2015). Aprobación del Acuerdo de París. FCCC/CP72015/L.9. New York: Naciones Unidas, Convención Marco sobre Cambio Climático. 
McAdam, J. (Mayo, 2011). Climate Change displacement and international law: complementary protection standards. Legal and protection policy research series. Ginebra: UNHCR.

McAdam, J. y Limon, M. (2015). Human Rights, climate change and cross-border displacemente: the role of the international human rights community in contributing to effective and just solutions. Ginebra: Universal Rights Group.

Podesta, J. y Ogden, P. (2007). The Security Implications of Climate Change. The Whashington Quarterly. Winter 2007-08. Boston: Center for Strategic and International Studies and the Massachusetts Institute of Technology.

Smith, D. y Vivekananda, J. (2007). A Climate of Conflict. The links between climate change, peace and war. Londres: International Alert, Printed by Jason Print.

The Guardian. (2015) Kiribati climate change refugee told he must leave New Zealand. Disponible en http://www.theguardian.com/environment/2015/sep/22/ kiribati-climate-change-refugee-told-he-must-leave-new-zealand

The Nansen Initiative (2015). The Protection Agenda. Disponible en www.thenanseninitiative.org

Türk, W. (Mayo, 2015). Temporary protection arrangements to fill a gap in the protection regime. Forced Migration Review, 49, 40-42.

UNHCR. (2014) UNHCR, the environment \& climate change. An overview. Ginebra: UNHCR.

UNFCCC. (2011). Informe de la Conferencia de las Partes sobre su $16^{\circ}$ período de sesiones, celebrado en Cancún del 29 de noviembre al 10 de diciembre de 2010. Disponible en http://unfccc.int/resource/docs/2010/cop16/spa/07a01s. pdf

Warner, K. (Mayo, 2011). Climate Change induced displacement: adaptation policy in the context of the UNFCCC climate change negotiations. Legal and protection policy research series. Ginebra: UNHCR. 